\title{
Resenha: Estudos sistêmico-funcionais no âmbito do Projeto SAL
}

\author{
Systemic Functional Studies in the SAL Project;
}

\author{
Ana Paula Carvalho Schmidt \\ Universidade Federal de Santa Maria - UFSM - Rio Grande do Sul - Brasil
}

\section{Jane Aparecida Florêncio}

Colégio Militar de Santa Maria - Rio Grande do Sul - Brasil

\begin{abstract}
Resumo: Estudos sistêmico-funcionais no âmbito do Projeto SAL (2018), organizado pelas linguistas Sara Regina Scotta Cabral, da Universidade Federal de Santa Maria (UFSM), e Leila Barbara, da Pontifícia Universidade Católica (SP) inclui investigações que enfocam temas pertencentes aos diferentes níveis de organização da linguagem, desde o estrato da léxico-gramática até o do contexto. Composto por 14 capítulos, aborda o contexto educacional, no âmbito escolar e acadêmico, com análises voltadas ao livro didático, à formação de professores em serviço e pré-serviço, à escrita acadêmica e à tradução. Outros contextos investigados são o literário, o midiático e o político.
\end{abstract}

Palavra-chave: Linguística Sistêmico-Funcional.

A linguagem em uso nos diferentes contextos é o objeto de investigação de pesquisadores do Projeto Sistêmica, Ambientes e Linguagens (SAL), inicialmente denominado Systemic Across Languages. Mais especificamente, no projeto, criado por Christian Matthiessen e Kasuhiro Teruya (Polythecnic University de Hong Kong) e por Leila Barbara (Pontifícia Universidade Católica de São Paulo - PUC/SP), com a participação de um grande grupo de pesquisadores latino-americanos, são desenvolvidos estudos essencialmente alicerçados em pressupostos teóricos da Linguística Sistêmico-Funcional (LSF). Nesse cenário, surge a obra denominada Estudos sistêmicofuncionais no âmbito do Projeto SAL (2018), livro sucessor de Estudos de Transitividade em linguística sistêmico-funcional (2018), ambos organizados pelas linguistas Sara Regina Scotta Cabral, da Universidade Federal de Santa Maria (UFSM), e Leila Barbara, da Pontifícia Universidade Católica (SP). Diferentemente do primeiro volume, em que o sistema de transitividade é basicamente colocado em foco, Estudos sistêmico- funcionais no âmbito do Projeto SAL amplia consideravelmente a dimensão linguística analisada, incluindo investigações que enfocam temas pertencentes aos diferentes níveis de organização da linguagem, desde o estrato da léxico-gramática até o do contexto.

Por um viés multimodal, este volume também se amplia, ao trazer uma investigação que versa sobre a linguagem não verbal. Entretanto, percebe-se que, apesar da ampliação, grande parte das pesquisas que investigam a linguagem verbal mobiliza a transitividade como um dos principais sistemas de análise. Isso acontece em 10 do total de 14 estudos reunidos neste compêndio, à exceção da introdução (na abertura do livro) e da apresentação dos autores (no fechamento). O contexto educacional, no âmbito escolar e acadêmico, é o mais pesquisado, com análises voltadas ao livro didático, à formação de professores em serviço e pré-serviço, à escrita acadêmica e à tradução. Outros contextos investigados são o literário, o midiático e o político. 
No contexto educacional, o livro didático é o objeto de investigação de três estudos do volume: Transitividade em estórias: orações materiais em instanciações dos gêneros exemplum e narrativa, de Fuzer e Gerhardt (Capítulo 1), La representación del mundo mediante los procesos del hacer y suceder, de Mizuno, Barletta e Chamorro (Capítulo 4) e O papel da cultura na coleção Formación en Español - lengua y cultura: um olhar sistêmico-funcional, de Mafessoni e Rottava (Capítulo 14). Os Capítulos 1 e 4 investigam as representações construídas por orações materiais: o primeiro, em livros de língua portuguesa e em um texto retirado do portal Domínio Público; o segundo, em livros escritos em espanhol para o ensino de Ciências Sociais e Naturais.

Enquanto Fuzer e Gerhardt centram sua análise nas etapas dos gêneros narrativa e exemplum, da família das estórias, Mizuno, Barletta e Chamorro preocupam-se com a representação de causalidade e agência em textos escolares colombianos. Por sua vez, no Capítulo 14, Mafessoni e Rottava apresentam a análise da coleção de língua espanhola Formación en Español - Lengua y Cultura (VILLALBA; GABARDO; MATA, 2012). Nesse estudo, as autoras analisam como o elemento cultural é tratado nos textos e qual sua relação com o aluno na obra.

O modelo transitivo da transitividade é mobilizado nos três referidos estudos. No Capítulo 4, emprega-se também 0 modelo ergativo da transitividade e, no Capítulo 14, o sistema de Modo (da metafunção interpessoal). Dessa forma, um mesmo objeto de pesquisa é escrutinado por meio de diferentes recortes que, quando reunidos, oferecem uma visão mais abrangente do fenômeno em discussão. Nos três estudos, sobressai-se a alta ocorrência de processos materiais. Para tais processos, são atribuídas as funções de a) representar ações ou acontecimentos causados por agentes externos para resolução da Complicação da narrativa (Capítulo 1), b) auxiliar na construção do mundo da experiência, tanto natural como social (Capítulo 4), e c) construir a representação do aluno como executor de ações solicitadas no enunciado (Capítulo 14). No que tange às práticas de leitura e escrita escolares, esses estudos contribuem para o mapeamento de gêneros para ler e escrever na escola. No caso específico do Capítulo 14, que investiga uma coleção de livros didáticos de língua espanhola, os resultados mostram que as obras dos sexto e nono anos possibilitam um maior acesso dos alunos aos modos de ser e pensar que os livros dos sétimo e oitavo anos.

Outras análises do contexto educacional presentes no volume têm como enfoque o professor em serviço - Representações de interdisciplinaridade construídas por professores em um programa de formação continuada, de Brum e Ticks (Capítulo 2), - e o professor pré-serviço - Processos mentais em portfólios de professores de inglês em formação inicial, de Vian Júnior e Ramalho (Capítulo 5). Brum e Ticks discutem as representações de interdisciplinaridade de professores de uma escola pública de Santa Maria/RS. Já Vian Júnior e Ramalho ocupam-se do mapeamento e da análise de processos mentais em portfólios reflexivos de estudantes de graduação em LetrasInglês. Para tanto, esses últimos valem-se de uma metodologia qualiquantitativa, com o auxílio do programa computacional WordSmith Tools (SCOTT, 2009). Ambos os estudos mobilizam a transitividade, porém, Brum e Ticks conciliam o complexo oracional à investigação. O ponto de familiaridade nas pesquisas é o processo reflexivo sobre a prática docente, por um lado, acerca do conceito de interdisciplinaridade, através de sessões de reflexão (Capítulo 2), e, por outro, em relação ao ensino e à aprendizagem da língua inglesa, através da escrita (Capítulo 5). As práticas relatadas contribuem para a formação docente.

A escrita acadêmica em dissertações e em artigos é também um objeto explorado na obra aqui resenhada, com auxílio do programa WordSmith Tools (SCOTT; TRIBBLE, 2006), ferramenta da Linguística de Corpus. Em Processos verbais e modalização na escrita acadêmica (Capítulo 6), Souza, Mendes e Penha analisam os sistemas de Modo e modalidade usados junto aos processos verbais dizer e afirmar em dissertações de mestrado da área de Linguística. Os processos verbais também são analisados em Um 
estudo tipológico dos processos verbais em um mesmo gênero: diferenças e semelhanças entre português e inglês (Capítulo 7), em que Joseph e Barbara caracterizam participantes e circunstâncias, juntamente com a detecção de padrões de organização da mensagem (citações, relatos e verbiagens) em artigos científicos em português e inglês das Ciências Agrárias. Ambas as pesquisas tratam de questões relacionadas às diferentes vozes (vozes externas ou do próprio autor) presentes nos textos. Por fim, os processos mentais, utilizados com o clítico "se", em artigos de diferentes áreas do conhecimento, são analisados em O clítico "se" em construções mentais com desfocamento de participantes (Capítulo 8). Morais explica as construções com desfocamento do participante Experienciador e articula pressupostos sistêmicofuncionais e da gramática tradicional para tratar de agência. Os achados dos Capítulos 6, 7 e 8 fornecem importantes subsídios para o ensino da escrita acadêmica.

O processo tradutório, no âmbito acadêmico, é abordado em $A$ prática tradutória a partir das noções de gênero e registro (Capítulo 10), em que Rottava e Hainzenreder discutem e apresentam uma proposta de estrutura potencial para a tradução como gênero discursivo. As autoras lançam a hipótese de que a constituição do que chamam de registro da tradução pode orientar as escolhas do tradutor ao oferecer uma descrição da configuração contextual dessa atividade social. O processo tradutório, no âmbito literário, por sua vez, é discutido em Estudos de tradução: transitividade na configuração de personagens no romance Harry Potter and the Chamber of Secrets $e$ sua tradução para o português brasileiro (Capítulo 9). Neste, Cruz faz uso da ferramenta WordSmith Tools para quantificar e destacar mudanças de processos verbais realizadas pela tradutora da obra, Lya Willer, que implicam em alterações nas representações das personagens construídas no texto-fonte. Essa dinâmica de tradução pode ser motivada por a) representações da língua de chegada, b) tentativa de evitar repetição de verbos de elocução e c) escolhas léxico-gramaticais da tradutora.
Assim como a investigação de Cruz, a discussão trazida por Representações em fábulas de Esopo e Millôr Fernandes (Capítulo 3), de Fuzer e Farencena, ilustra como gêneros literários também podem constituir objeto de análise da LSF. Com foco no gênero fábula, as autoras analisam representações das personagens a partir da integração de categorias dos sistemas de transitividade e avaliatividade, e revelam que as posições dos participantes invertem-se em termos de agência, já que oscilam entre o papel de agente e o de paciente. Uma vez comparados os capítulos de Cruz (Capítulo 9) e Fuzer e Farencena (Capítulo 3), percebe-se que o primeiro possibilita inferir a existência de questões culturais subjacentes, como a influência do contexto de circulação do texto traduzido nas escolhas léxico-gramaticais tradutórias, enquanto o segundo revela questões ideológicas, como as relações de poder vivenciadas na época.

Textos midiáticos verbais e não verbais são analisados em Avaliatividade: marcas linguísticas em exemplares do gênero exposição (Capítulo 11), de Freitas e Cabral, e Avaliatividade em imagens (Capítulo 12), de Florek. Em Freitas e Cabral, o foco está na verificação da função semântica de mecanismos predominantes em textos veiculados na página Observatório de Imprensa sobre a Operação Lava Jato. Por sua vez, Florek, tomando por base a Gramática do Design Visual, de Kress e Van Leeuwen (2006), propõe categorias gerais para itens avaliativos presentes no modo semiótico visual em imagens jornalísticas. Os resultados apresentados nesses capítulos compartilham descobertas que se assemelham e, ao mesmo tempo, distinguem-se. Em ambos há a predominância de mecanismos dos subsistemas de Atitude; entretanto, o Capítulo 11 está voltado para a Apreciação, e o Capítulo 12, para o Afeto. Textos do contexto midiático podem se constituir em poderosos recursos didáticos para o ensino de marcas avaliativas que, consequentemente, têm potencial para fomentar o letramento crítico no ensino de leitura e escrita.

O discurso político é examinado em The place of the concept of hegemony in political discourse analysis (PDA): a tentative link in the light of critical 
discourse analysis (CDA) and Systemic Functional Grammar (SFG) (Capítulo 13), autorado por De Souza. A pesquisa busca responder a em que medida os discursos em epístolas de Padre Cícero reproduzem ou desafiam as configurações hegemônicas de seu tempo. Para isso, o autor conta com pressupostos teóricos da Análise Crítica do Discurso (ACD) (FAIRCLOUGH, 1989; 1995) e da Gramática Sistêmico-Funcional (GSF) (HALLIDAY, 1961; 1967a; 1967b; 1968; 1970). De Souza conclui que Padre Cícero aparentemente questionava as relações de poder de sua época, entretanto seu discurso harmonizava as relações entre seus pares políticos. Como consequência, Juazeiro tornou-se independente.

Assim como o Capítulo 13, os Capítulos 2 e 12 entrelaçam conceitos de domínios distintos do conhecimento - da Sociologia (por exemplo, GRAMSCI, 1971; LACLAU; MOUFFE, 1985; BERNSTEIN, 1996) e da Linguística (por exemplo, FAIRCLOUGH, 1995; CELANI; MAGALHÃES, 2002; HALLIDAY; MATTHIESSEN, 2004). Na área da Linguística, também se encontra, em outros capítulos do volume, diversidade na adoção de enquadres teóricos de pesquisa, segundo diferentes abordagens aos estudos da linguagem: Formação de Professores (por exemplo, CELANI; MAGALHÃES, 2002; GIMENEZ, 2005), Estudos da Tradução (por exemplo, HOUSE, 1997; MATTHIESSEN, 2001; JESUS, 2012), Análise Crítica do Discurso (por exemplo, VAN DIJK, 1991; FAIRCLOUGH, 1995; MOTTA-ROTH, 2006) e Gramática (por exemplo, MONTEIRO, 1994; BAGNO, 2000). Na perspectiva sistêmico-funcional, têm-se, para o estudo de gêneros, a Pedagogia de Gêneros (por exemplo, ROSE; MARTIN; 2012) e a Estrutura Potencial de Gênero (por exemplo, HASAN, 1985); para o modo semiótico não verbal, a Gramática do Design Visual (KRESS; VAN LEEUWEN, 2006). Posto isso, evidencia-se, nesta coletânea, um profícuo diálogo, dentro da própria LSF e também com outras disciplinas, disponibilizando vasto material para subsidiar práticas de ensino e aprendizagem de leitura e escrita para variados contextos educacionais.
Em seu conjunto, a obra pode ser considerada acessível a uma parte específica do público que integra a Linguística Aplicada e áreas afins. Estudantes de graduação que tenham algum conhecimento prévio da teoria podem se servir dos estudos que, sucintamente, revisitam conceitos fundamentais da LSF. Estudantes de pós-graduação podem se beneficiar da oferta de diversificados percursos analíticos, propostos a partir de diferentes sistemas das metafunções, em variados gêneros, da ordem do estrato linguístico e extralinguístico. Não obstante, o referido público também pode se aprofundar em determinados temas discutidos, pautando-se no amplo campo de pesquisas já desenvolvidas, apresentadas por alguns estudos.

O volume, que agrega estudos latinoamericanos, carece de um número maior de pesquisas acerca de outros contextos que não o brasileiro. Apenas textos do contexto colombiano são objeto de exame em uma das pesquisas (Capítulo 4). Seria interessante que houvesse mais pesquisas, do Brasil ou de outros países, escritas em outras línguas, assim, podendo estabelecer um diálogo com comunidades que não falam português ou espanhol. As oportunidades de intercâmbio de pesquisa provavelmente seriam bem maiores. Para se ter uma ideia, na coletânea, apenas um capítulo, de uma pesquisa desenvolvida no contexto brasileiro, é escrito em língua inglesa (Capítulo 13). Por outro lado, isso já é um aceno para que, talvez, em um próximo volume, sejam congregados trabalhos em outras línguas, do Brasil ou de outros países latino-americanos.

Uma vantagem desta obra é a sua disponibilização gratuita, em formato de ebook, na página https://www.ufsm.br/laboratorios/labport/. Desse modo, um número expressivo de leitores pode ter acesso a tal profícuo diálogo linguístico. Certamente, tal conjunto de pesquisas pode reverberar e alcançar outros espaços de pesquisa e, desse modo, fomentar a cooperação interinstitucional, ao possibilitar a identificação de interesses de pesquisa semelhantes, voltadas para um mesmo gênero ou contexto. 


\section{REFERÊNCIAS}

BAGNO, Marcos. Dramática da Língua Portuguesa: tradição gramatical, mídia e exclusão social. São Paulo: Loyola, 2000. 327 p.

BERNSTEIN, Basil. A estruturação do discurso pedagógico: classe, códigos e controle. Petrópolis: Vozes, 1996. 307 p.

CABRAL, Sara Scotta; BARBARA, Leila. (Orgs.). Estudos de transitividade em linguística sistêmico-funcional. Santa Maria: UFSM, PPGL, 2018. 290 p.

CELANI, Maria Antonieta Alba; MAGALHÃES, Maria Cecília Camargo. Representações de professores de inglês como língua estrangeira sobre suas identidades profissionais: uma proposta de reconstrução. In: MOITA LOPES, Luiz Paulo da; BASTOS, Liliana Cabral (Orgs.). Identidades: recortes multi e interdisciplinares. Campinas: Mercado de Letras, 2002. p. 319-338.

FAIRCLOUGH, Norman. Language and power. London: Longman, 1989. 259 p.

Critical Discourse analysis: the critical study of language. London: Longman, 1995. 265 p.

GIMENEZ, Telma. Desafios contemporâneos na formação de professores de línguas: contribuições da linguística aplicada. In: FREIRE, Maximina Maria; ABRAÃO, Maria Helena Vieira; BARCELOS, Ana Maria Ferreira. Linguística aplicada e contemporaneidade. São Paulo: Pontes Editores, 2005. p. 183-201.

GRAMSCI, Antonio. Selections from the Prison Notebooks. New York: International Publishers Co., 1971.

HALLIDAY, Michael Alexander Kirkwood. Categories of the theory of grammar. In: WEBSTER, Jonathan James (Org.) On Grammar. Collected works of M. A. K. Halliday, v.1. London/New York: Continuum, 1961 [2002], p. 37-94.

Notes on transitivity in English: Part 1. Journal of linguistics, v. 3, n. 1, p. 37-81, 1967a.

Notes on transitivity in English: Part 2. Journal of linguistics, v. 3, n. 2, p. 199-244, 1967b.

Notes on transitivity in English: Part 3. Journal of linguistics, v. 4, n. 2, p. 179-215, 1968.

Functional diversity in language as seen from a consideration of modality and mood in English. Foundations of Language: International Journal of Language and Philosophy, v. 6, n. 3, p. 322-361, 1970.

HALLIDAY, Michael Alexander Kirkwood; MATTHIESSEN, Christian Mathias Ingemar Martin. An introduction to functional grammar. 3. ed. London, New York: Arnold, 2004.

HASAN, Ruqaiya. Part B. In: HALLIDAY, Michael Alexander Kirkwood; HASAN, Ruqaiya. Language, context, and text: aspects of language in a social-semiotic perspective. Oxford: Oxford University Press, 1985, p. 52121.
HOUSE, Juliane. Translation quality assessment: a model revisited. Tubingen: Niemeyer, 1997.

JESUS, Silvana Maria de. Estudos sistêmico-funcionais da tradução. Domínios de Lingu@gem, v. 6, n. 1, p. 286301,2012

KRESS, Gunther; VAN LEEUWEN, Theo. Reading images: the grammar of the design visual. London: Routledge, 2006. 321 p.

LACLAU, Ernesto; MOUFFE, Chantal. Hegemony and socialist strategy. London/New York: Verso, 1985. 205 p.

MATTHIESSEN, Christian Mathias Ingemar Martin. The environments of translation. In: STEINER, Erich; YALLOP, Colin. (Orgs.). Exploring translation and multilingual text production: beyond content. Berlin: Mouton de Gruyter, 2001, p. 41-124.

MONTEIRO, José Lemos. A questão do se. In: MONTEIRO, José Lemos. Pronomes pessoais: subsídios para uma gramática do português do Brasil. Fortaleza: EUFC, 1994.

MOTTA-ROTH, Desirée. Competências comunicativas interculturais no ensino de inglês como língua estrangeira. In: MOTTA-ROTH, Desirée; BARROS, Nina Célia; RICHTER, Marcos. (Orgs.). Linguagem, cultura e sociedade. Santa Maria: PPGL Editores/UFSM, 2006, p. 191-201.

ROSE, David; MARTIN, James Robert. Learning to write, Reading to learn: genre, knowledge and pedagogy in the Sydney School. London: Equinox, 2012.

SCOTT, Mike. Wordsmith Tools (versão 5.0). Lexical analysis software. Liverpool, 2009.

SCOTT, Mike; TRIBBLE, Christopher. Studies in Corpus Linguistics: textual patterns. Amsterdam/Philadelphia: John Benjamins Publishing Co., 2006.

VAN DIJK, Teun. Racism and the press. London: Routledge, 1991. 276 p.

VILLALBA, Terumi Koto Bonnet; GABARDO, Maristella; MATA, Rodrigo Rodolfo Ruibal. Formación en Español: lengua y cultura - Descripción. Curitiba: Base Editorial LTDA. 2012. $168 \mathrm{p}$.

\section{COMO CITAR ESSE ARTIGO}

CARVALHO SCHMIDT, Ana Paula; FLORÊNCIO, Jane Aparecida. Estudos sistêmico-funcionais no âmbito do Projeto SAL. Signo, Santa Cruz do Sul, v. 46, n. 86, maio 2021. ISSN 1982-2014. Disponível em: $<$ https://online.unisc.br/seer/index.php/signo/article/view/1590 5>. doi:https://doi.org/10.17058/signo.v46i86.15905. 